\title{
Dynamical Band Flipping in Fermionic Lattice Systems: An ac-Field-Driven Change of the Interaction from Repulsive to Attractive
}

\author{
Naoto Tsuji, ${ }^{1}$ Takashi Oka, ${ }^{1}$ Philipp Werner, ${ }^{2}$ and Hideo Aoki ${ }^{1}$ \\ ${ }^{1}$ Department of Physics, University of Tokyo, Hongo, Tokyo 113-0033, Japan \\ ${ }^{2}$ Theoretical Physics, ETH Zurich, 8093 Zürich, Switzerland
}

(Received 16 August 2010; revised manuscript received 1 February 2011; published 6 June 2011)

\begin{abstract}
We show theoretically that the sudden application of an appropriate ac field to correlated lattice fermions flips the band structure and effectively switches the interaction from repulsive to attractive. The nonadiabatically driven system is characterized by a negative temperature with a population inversion. We numerically demonstrate the converted interaction in an ac-driven Hubbard model with the nonequilibrium dynamical mean-field theory solved by the continuous-time quantum Monte Carlo method. Based on this, we propose an efficient ramp-up protocol for ac fields that can suppress heating, which leads to an effectively attractive Hubbard model with a temperature below the superconducting transition temperature of the equilibrium system.
\end{abstract}

DOI: 10.1103/PhysRevLett.106.236401

PACS numbers: 71.10.Fd, 03.65.Vf, 03.75.Ss

Introduction.-There is an increasing fascination with the dynamics of fermions driven by external fields as a result of recent developments in time-resolved experimental techniques in, e.g., ultracold atom physics [1] and electrons in a crystal [2]. There, the external fields are employed not only for probing the response against perturbation, but also for creating excited states to control phases of the system. In particular, continuously driven systems isolated from the environment exhibit nonequilibrium statistical distributions that dramatically alter their physical properties. One basic, long-known example is the negative temperature $(T)$ distribution [3], where higher energy levels are occupied (population inversion). If such distributions are realized in correlated fermion systems, it should have a huge impact on many-body physics since, as we shall show, the originally repulsive interfermion interaction effectively turns into an attractive one as a result of the negative $T$.

To start with, a realization of negative $T$ requires as a crucial condition that the energy spectrum is upper bounded [3]. A spin system, for which the concept of negative $T$ was originally introduced [4], satisfies this condition. Another promising candidate for negative $T$ is a many-particle system on a lattice, where particles form a band structure with a finite bandwidth, as far as the interband excitations induced by external fields are forbidden or negligible. For instance, Ref. [5] proposes that a harmonic potential trapping bosonic atoms in an optical lattice can be inverted to realize a negative $T$.

In the present Letter, we take a totally different approach, namely, a sudden application (quench) of a sinusoidal force (ac field) to a lattice fermion system. The effect of ac fields has been discussed previously in the context of adiabatic processes (e.g., [6]), where the hopping amplitude is effectively renormalized [7]. Here we show that the "nonadiabatic" switch-on of the ac field can dynamically invert the band structure (dynamical band flipping), generating a negative- $T$ distribution. Based on this, we suggest that the ac quench is related to an interaction quench ("ac/ $U$-quench correspondence"), where the interaction can be effectively converted between repulsive and attractive. This is numerically demonstrated for the ac-driven Hubbard model, whose time evolution is obtained with the nonequilibrium dynamical mean-field theory (DMFT) [8,9] solved with the continuous-time quantum Monte Carlo (QMC) method [10].

One immediate question may be whether the sudden switch-on of the ac field causes a violent heating of the system. However, we shall show that the heating associated with the sign change of the interaction can be suppressed if the ac field is turned on in a suitable way. Therefore, if the ac-driven system thermalizes, it will correspond to an effectively attractive Hubbard model whose temperature is low enough that the system can possibly accommodate superconductivity.

Formulation.-We consider a model Hamiltonian,

$$
\mathcal{H}(t)=-J \mathcal{H}_{K}+U \mathcal{H}_{I}+\cos (\Omega t) \sum_{j, \sigma} \boldsymbol{K}(t) \cdot \boldsymbol{R}_{j} n_{j \sigma}
$$

with $\quad \mathcal{H}_{K}=\sum_{\langle i j\rangle, \sigma}\left(c_{i \sigma}^{\dagger} c_{j \sigma}+\right.$ H.c. $) \quad$ and $\quad \mathcal{H}_{I}=$ $\sum_{j}\left(n_{j \uparrow}-\frac{1}{2}\right)\left(n_{j \downarrow}-\frac{1}{2}\right)$. Here $c_{j \sigma}^{\dagger}$ creates a fermion at site $j$ with spin $\sigma$ for electron systems or pseudospin $\sigma$ for cold atoms, $\boldsymbol{R}_{j}$ is a position vector, $n_{j \sigma}=c_{j \sigma}^{\dagger} c_{j \sigma}, J(>0)$ the hopping energy, $U(>0)$ the on-site interaction. The third term in Eq. (1) represents the ac field with amplitude $\boldsymbol{K}$ and frequency $\Omega$. We take, for the DMFT, a $d$-dimensional hypercubic lattice with $\boldsymbol{K}(t)=K(t)(1,1, \ldots, 1)$, and consider a half-filled band. We assume that the system is initially in equilibrium at temperature $T$, and the ac field is suddenly switched on at $t=0$, i.e., $K(t)=K \theta(t)$ with $\theta(t)$ the step function. 
It has been shown [7], for noninteracting systems, that the effect of the ac field is simply a renormalization of the hopping energy,

$$
J \rightarrow J_{\text {eff }}=\mathcal{J}_{0}(K / \Omega) J,
$$

with $\mathcal{J}_{n}(z)$ the $n$th order Bessel function. A naive explanation of Eq. (2) is that in the presence of the ac field the original band dispersion $\epsilon_{k}=-2 J \sum_{i=1}^{d} \cos k_{i}$ is replaced with a time-averaged $\bar{\epsilon}_{k}=\frac{\Omega}{2 \pi} \int_{0}^{2 \pi / \Omega} d t \epsilon_{k-A(t)}=$ $\mathcal{J}_{0}(K / \Omega) \epsilon_{k}$, where we take a gauge in which the ac field is represented by a vector potential $\boldsymbol{A}(t)=$ $-\boldsymbol{K} \sin (\Omega t) / \Omega$. The renormalization of $J$ can more rigorously be derived from the Floquet theory for ac fields [11]. Curiously, $J_{\text {eff }} \propto \mathcal{J}_{0}(K / \Omega)$ then changes sign as $\mathcal{A} \equiv K / \Omega$ is increased. This implies that $J_{\text {eff }}$ vanishes at $\mathcal{A}_{1}=2.404 \ldots$ (see the inset of Fig. 3), which is known as dynamical localization [7]. Experimentally, the scaling (2) was beautifully observed in a Bose-Einstein condensate (BEC) in an optical lattice [12].

What happens when $\mathcal{J}_{0}(\mathcal{A})<0$ ? In equilibrium, the inverted sign of $J$ does not change the physics, since it can be canceled with a gauge transformation $c_{j \sigma}^{\dagger} \rightarrow-c_{j \sigma}^{\dagger}$ for the $j \in B$ sublattice. Out of equilibrium, however, we have to consider the distribution of occupied states, and the physics can indeed dramatically change as we cross the zeros of $\mathcal{J}_{0}(\mathcal{A})$.

To characterize nonequilibrium distributions, we can define a time-resolved spectral function, $A(\omega, t) \equiv$ $-\frac{1}{\pi} \operatorname{Im} \int d t^{\prime} e^{i \omega t^{\prime}} \sum_{k} G_{k \sigma}^{R}\left(t+\frac{t^{\prime}}{2}, t-\frac{t^{\prime}}{2}\right)$ and an occupation $N(\omega, t) \equiv-\frac{i}{2 \pi} \int d t^{\prime} e^{i \omega t^{\prime}} \sum_{k} G_{k \sigma}^{<}\left(t+\frac{t^{\prime}}{2}, t-\frac{t^{\prime}}{2}\right)$, in terms of the retarded $G_{k \sigma}^{R}\left(t, t^{\prime}\right)=-i \theta\left(t-t^{\prime}\right)\left\langle\left\{c_{k \sigma}(t), c_{k \sigma}^{\dagger}\left(t^{\prime}\right)\right\}\right\rangle$ and lesser Green function $G_{k \sigma}^{<}\left(t, t^{\prime}\right)=i\left\langle c_{k \sigma}^{\dagger}(t) c_{k \sigma}\left(t^{\prime}\right)\right\rangle$. For noninteracting fermions we can evaluate [11], for $\Omega \gg J$, the longtime behavior as

$$
\begin{gathered}
A(\omega, t) \stackrel{t \rightarrow \infty}{\longrightarrow} \frac{1}{\left|\mathcal{J}_{0}(\mathcal{A})\right|} \rho_{0}\left(\frac{\omega}{\left|\mathcal{J}_{0}(\mathcal{A})\right|},\right. \\
N(\omega, t) \stackrel{t \rightarrow \infty}{\longrightarrow} A(\omega, \infty) f_{T}\left(\frac{\omega}{\mathcal{J}_{0}(\mathcal{A})}\right),
\end{gathered}
$$

where $\rho_{0}(\omega)=\sum_{k} \delta\left(\omega-\epsilon_{k}\right)$ is the noninteracting density of states, $f_{T}(\omega)=1 /\left(e^{\omega / T}+1\right)$ the Fermi distribution with $k_{B}=1$, and we have used the symmetry $\rho_{0}(-\omega)=$ $\rho_{0}(\omega)$. We notice that the sign of $\mathcal{J}_{0}(\mathcal{A})$ is irrelevant to the spectrum, but it is indeed relevant to the occupation. In fact, for $\mathcal{J}_{0}(\mathcal{A})<0$ the effective temperature is negative, since $f_{T}\left(\omega / \mathcal{J}_{0}(\mathcal{A})\right)=f_{-T}\left(\omega /\left|\mathcal{J}_{0}(\mathcal{A})\right|\right)$. One intuitive way to interpret this is that, as the band is flipped when $J$ changes sign due to the ac field, each fermion follows the change with no shift in the momentum (Fig. 1 for $T<0$ ). This is the essential mechanism for the negative $T$ distribution that we propose here.

Interacting systems driven by ac fields have also been theoretically studied for the Bose-Hubbard

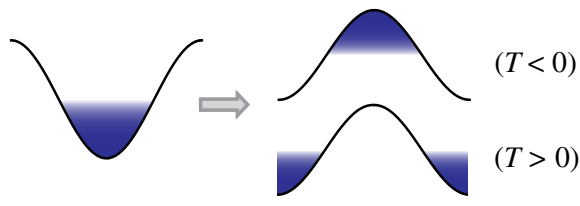

FIG. 1 (color online). A schematic band flipping accompanied with a population inversion $(T<0)$ induced by the ac field when $\mathcal{J}_{0}(\mathcal{A})<0$ and without a population inversion $(T>0)$.

model $[6,13]$, and the Falicov-Kimball model [11,14]. Let us assume that $J$ is suddenly quenched by the ac field as in Eq. (2). Then the density matrix timeevolves as $\left.\left.\rho(t)=e^{-i t\left(-J_{\text {eff }}\right.} \mathcal{H}_{K}+U \mathcal{H}_{I}\right) \rho(0) e^{i t\left(-J_{\text {eff }}\right.} \mathcal{H}_{K}+U \mathcal{H}_{I}\right)=$ $e^{\mp i \tilde{t}\left(-J \mathcal{H}_{K}+U_{\text {eff }} \mathcal{H}_{I}\right)} \rho(0) e^{ \pm i \tilde{t}\left(-J \mathcal{H}_{K}+U_{\text {eff }} \mathcal{H}_{I}\right)} \equiv \rho_{\mathcal{A}}^{ \pm}(\tilde{t})$, where the upper (lower) sign corresponds to $\mathcal{J}_{0}(\mathcal{A})>(<) 0$, and time is rescaled as $\tilde{t}=\left|\mathcal{J}_{0}(\mathcal{A})\right| t$. After that, the $J$ quench is translated to the interaction quench [15] as

$$
U \rightarrow U_{\text {eff }}=U / \mathcal{J}_{0}(\mathcal{A}) .
$$

For $\mathcal{J}_{0}(\mathcal{A})<0$, the corresponding $U$ quench evolves in time with $\rho_{\mathcal{A}}^{-}(\tilde{t})$ where the phase rotates in the reverse direction. We can relate $\rho_{\mathcal{A}}^{-}(\tilde{t})$ with the normal time evolution $\rho_{\mathcal{A}}^{+}(\tilde{t})$ via an energy reversal; i.e., the signs of all the quantities (except for time) that have dimension of energy are reversed. For example, energy reversal maps kinetic energy $E_{\text {kin }}(t) \equiv-i \sum_{k} \epsilon_{k-A(t)} G_{k \sigma}^{<}(t, t)$ to $-E_{\text {kin }}(t)$. We summarize the relation between ac quench and $U$ quench in Fig. 2.

The ac/U-quench correspondence, which we numerically demonstrate in the following, has the intriguing consequence that for $\mathcal{J}_{0}(\mathcal{A})<0$ the ac field effectively switches the many-body interaction from repulsive to attractive $\left(U_{\text {eff }}<0\right)$ (5). In addition, it implies that the system thermalizes to a negative-temperature canonical distribution: according to the thermalization hypothesis $[15,16]$, the (nonintegrable) Hubbard model evolving with normal $\rho_{\mathcal{A}}^{+}(\tilde{t})$ thermalizes after a $U$ quench in the long-time limit with a positive temperature $T_{\text {eff }}(>0)$. Since $\rho_{\mathcal{A}}^{-}(\tilde{t})$ is related to $\rho_{\mathcal{A}}^{+}(\tilde{t})$ via the energy reversal (Fig. 2), the ac/U-quench correspondence implies that the system driven by the ac field should finally thermalize with a negative temperature, $-T_{\text {eff }} /\left|\mathcal{J}_{0}(\mathcal{A})\right|$.

Numerical result.-To verify the interaction switching implied by the ac/U-quench correspondence in the dynamical band-flipping in interacting systems, we have numerically computed the time evolution of the system using the nonequilibrium DMFT [9], which can treat the

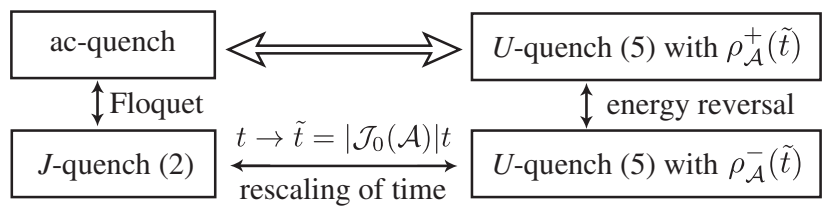

FIG. 2. The ac/U-quench correspondence. 


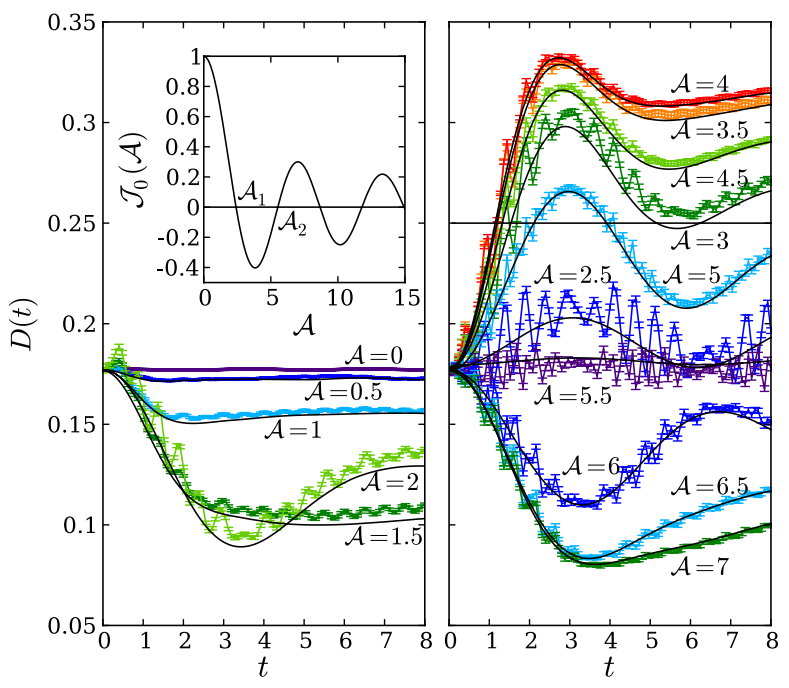

FIG. 3 (color online). Time evolution of the double occupancy $D$ for various values of $\mathcal{A} \equiv K / \Omega$ (symbols with error bars) for $\Omega=2 \pi, U=1$, with the horizontal line indicating the freeelectron value of $D=0.25$. Solid curves represent the corresponding result for the $U$ quench with $\rho_{\mathcal{A}}^{+}(\tilde{t})$ plotted on a rescaled time axis $t=\tilde{t} /\left|\mathcal{J}_{0}(\mathcal{A})\right|$. Inset depicts the Bessel function, $\mathcal{J}_{0}(\mathcal{A})$.

dynamics of infinite systems with the local correlations from the many-body interactions fully taken into account. In the DMFT calculation, we restrict ourselves to paramagnetic phases without charge order. The hopping is scaled with dimension $d$ as $J=J^{*} / 2 \sqrt{d}(d \rightarrow \infty)$ [8]. In the following we take $J^{*}$ as the unit of energy and write $J^{*}$ as $J$. Even when fermions are initially weakly interacting, the effective interaction (5) plunges into the strongcoupling regime around the zeros of $\mathcal{J}_{0}(\mathcal{A})$, so that we need an impurity solver that is valid for both weak and strong interactions. Here we employ the continuous-time QMC method based on a weak-coupling expansion [10], which is numerically exact within statistical errors.

A simple measure of whether the interaction is repulsive or attractive is the double occupancy, $D(t)=\left\langle n_{j \uparrow}(t) n_{j \downarrow}(t)\right\rangle$. Figure 3 displays the time evolution of the double occupancy for various amplitudes $\mathcal{A}=K / \Omega$ with fixed $\Omega=2 \pi, U=1$, and $T=0.1 . D(t)$ starts from the equilibrium value, which is, for the repulsive interaction, smaller than the noninteracting value, $\left\langle n_{j \uparrow}\right\rangle\left\langle n_{j \downarrow}\right\rangle=0.25$. For $\mathcal{A}<\mathcal{A}_{1}$ (left panel of Fig. 3), $D(t)$ decreases with $t$, which is natural since the effective interaction (5) is enhanced. For large effective interaction, the double occupancy shows $2 \pi / U$-periodic collapse-and-revival oscillations [15], while the fast oscillations with frequency $2 \Omega$ come from a nonlinear effect of the ac field.

The double occupancy starts to behave in a dramatically different manner as we plunge into the $\mathcal{J}_{0}(\mathcal{A})<0$ regime, i.e., $\mathcal{A}_{1}<\mathcal{A}<\mathcal{A}_{2}=5.520 \ldots$ (right panel of Fig. 3): $D(t)$ steeply increases after the ac field is switched on, and even goes "beyond" the noninteracting value 0.25 .
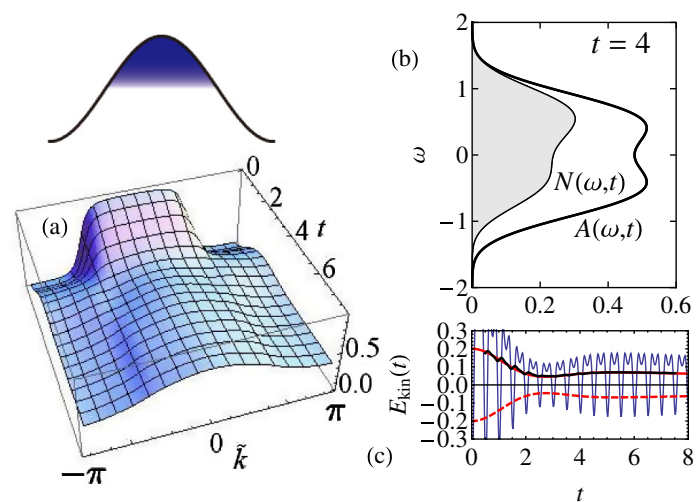

FIG. 4 (color online). (a) The momentum distribution, (b) the spectral function (thick curve), the occupation (shaded region), and (c) the kinetic energy for an ac quench with $\mathcal{A}=4$, $\Omega=2 \pi$, and $U=1$ (oscillatory curve, along with a timeaverage in black), as compared with the corresponding $U$ quench (dashed curve, along with a sign-inverted plot). The top left inset schematically shows a negative- $T$ situation.

This implies that fermions prefer a large double occupancy, evidence that the many-body interaction indeed turns into an attraction. When $\mathcal{J}_{0}(\mathcal{A})$ returns to positive for $\mathcal{A}>\mathcal{A}_{2}, D(t)$ again becomes smaller than 0.25 , and the effective interaction goes back to repulsive.

To endorse the ac/U-quench correspondence (Fig. 2) quantitatively, we plot $D(t)$ [solid curves in Fig. 3, plotted as a function of the rescaled time $\left.t=\tilde{t} /\left|\mathcal{J}_{0}(\mathcal{A})\right|\right]$ and $E_{\text {kin }}(t)$ [dashed curve in Fig. 4(c)] for the $U$ quench calculated with $\rho_{\mathcal{A}}^{+}(\tilde{t})$. For $D(t)$, we can see that the $U$ quench results agree with those for the (nonoscillatory components of) the ac quenches to a surprisingly good accuracy. The accuracy is especially excellent for $\mathcal{A} \gtrsim 4$, while for $\mathcal{A} \lesssim 3$ the small differences seen between the $U$ and ac quench come from the fact that it takes a few cycles for the ac field to renormalize $J$. For $\mathcal{J}_{0}(\mathcal{A})<0$ we again confirm the ac/U-quench correspondence in the kinetic energy if we consider the energy reversal $E_{\text {kin }}(t) \rightarrow-E_{\text {kin }}(t)$ in the $U$ quench [a sign-inverted plot of the dashed curve in Fig. 4(c)] and time-average $E_{\text {kin }}(t)$ over one cycle in the ac quench (black curve).

As for the population inversion, we can also obtain its direct evidence by calculating the (gauge-invariant) momentum distribution function [11] $f(\tilde{\boldsymbol{k}}, t) \equiv-i G_{\tilde{\boldsymbol{k}}}^{<}(t, t)$ $(\tilde{\boldsymbol{k}}(t)=\boldsymbol{k}+\boldsymbol{A}(t))$ in Fig. 4(a), and $A(\omega, t)$ and $N(\omega, t)$ [17] in Fig. 4(b) for the same $U, \Omega$, and $T$ as in Fig. 3, with $\mathcal{A}=4$ [for which $\mathcal{J}_{0}(\mathcal{A})<0$ ]. We plot $f(\tilde{\boldsymbol{k}}, t)$ along a typical slice [18] in the Brillouin zone. In the initial state, the particles distribute around $-\frac{\pi}{2}<\tilde{k}<\frac{\pi}{2}$ with a blurred Fermi surface. As the ac field is turned on, the distribution is gradually smeared out, but the main population continues to stick to $-\frac{\pi}{2}<\tilde{k}<\frac{\pi}{2}$. This sharply contrasts with what has been observed in BECs [12]: there, the system adiabatically follows the lowest energy states with no population inversion (Fig. 1 for $T>0$ ) with the peak of 
$f(\tilde{k}, t)$ shifted by $\pi$ in the flipped band [19]. However, our results indicate that, with sufficiently fast ramp up of the ac field in fermion systems, the system does not relax to lower energy states, but the population can be inverted. The population inversion is more directly seen in $N(\omega, t)$ [Fig. 4(b)], where fermions tend to occupy higher energy states $(\omega>0)$, justifying our picture (Fig. 1 for $T<0$ ). This kind of drastic change of the dynamics as a function of the parameter is reminiscent of phenomena observed in nonlinear mechanics. It may thus be interesting to try to connect bifurcation theory [20] to the band-flipping phenomena found here.

Suppression of heating - Now that the ac field is found to effectively convert a repulsive interaction into an attraction, one may contemplate the possibility of ac-induced superconductivity (SC), assuming that the system thermalizes. The obvious interest is that it is far easier to realize SC in attractive systems with $s$-wave pairing than in repulsive ones where the SC gap has to be anisotropic, so that the ac drive may provide an advantageous as well as novel avenue for SC. However, a simple, sudden quench would heat the system to, e.g., $\left|T_{\text {eff }} / J_{\text {eff }}\right|=1.83 \pm 0.02$ for $\mathcal{A}=4$, $\Omega=2 \pi$, and $U=1$ (Fig. 4), which is much larger than the critical temperature for the attractive Hubbard model $\left(T_{c} / J \sim 0.1\right.$ for $U \sim-2$ [21]). So the crucial question is whether we can avoid such a heating.

A smooth change in $U_{\text {eff }}$ from positive to negative might seem desirable, but this is unfortunately impossible, since $\left|U_{\text {eff }} / U\right|$ has to cross a singularity at $\left|\mathcal{J}_{0}(\mathcal{A})\right|=0$. Instead, we propose here a "multistep quench" (an example is shown in the left panel of Fig. 5). (i) We start from a weak interaction. (ii) This is followed by a sudden quench (to avoid the above problem) to an attractive but still weak interaction. The reason we start from a small $U$ is that a larger jump of $U_{\text {eff }}$ in a sudden quench tends to cause a higher temperature. (iii) After the sudden quench we employ a smooth ramp to amplify $U_{\text {eff }}$.

In the right panel of Fig. 5 we plot $T_{\text {eff }}(\tilde{t})$ for one example of the multistep $U$ quench $\left(U_{\text {eff }}=0.2 \rightarrow\right.$ $-0.5 \rightarrow-2)$, which is estimated by equating the total energy of the system at each rescaled $\tilde{t}$ with the one for an equilibrium system with temperature $\tilde{T}$. For the example displayed here $\left|T_{\text {eff }}(\tilde{t}=5) / J_{\text {eff }}\right|=0.080 \pm 0.004$ with an initial $T / J=0.05$, which accomplishes a temperature lower than $T_{c} / J \sim 0.1$.

Finally, we mention the stability and experimental feasibility of the population inversion. Once the negative- $T$ distribution is realized, it does not relax to a lower energy state due to energy conservation as long as the system is isolated from the environment with no energy dissipation. If fermions are coupled to other degrees of freedom, the distribution should start to collapse to the normal one with a positive $T$ with a decay rate determined by the strength of the coupling to the environment. One good candidate that can avoid this difficulty is a system of cold fermionic atoms
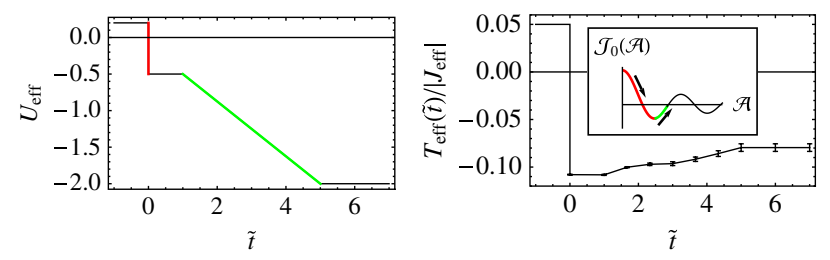

FIG. 5 (color online). An example of a multistep quench (left panel), and the corresponding evolution of $T_{\text {eff }}(\tilde{t})$ (right). The inset indicates the path for the ac quench on $\mathcal{J}_{0}(\mathcal{A})$.

trapped in an optical lattice, which is a virtually ideal, isolated single-band system [5]. There, a conversion from attractive to repulsive interactions, which is equally feasible, may also be interesting. For electron systems, possible candidates are superlattices or arrays of nanostructures designed to realize a single-band well separated from other bands.

We acknowledge illuminating discussions with $\mathbf{M}$. Eckstein, T. Esslinger, and L. Tarruell. N. T. was supported by the Japan Society for the Promotion of Science.

[1] I. Bloch, J. Dalibard, and W. Zwerger, Rev. Mod. Phys. 80, 885 (2008).

[2] A. L. Cavalieri et al., Nature (London) 449, 1029 (2007).

[3] N. F. Ramsey, Phys. Rev. 103, 20 (1956); M. J. Klein, ibid. 104, 589 (1956).

[4] E. M. Purcell and R. V. Pound, Phys. Rev. 81, 279 (1951).

[5] A. P. Mosk, Phys. Rev. Lett. 95, 040403 (2005).

[6] A. Eckardt, C. Weiss, and M. Holthaus, Phys. Rev. Lett. 95, 260404 (2005).

[7] D. H. Dunlap and V. M. Kenkre, Phys. Rev. B 34, 3625 (1986); M. Holthaus, Phys. Rev. Lett. 69, 351 (1992).

[8] A. Georges et al., Rev. Mod. Phys. 68, 13 (1996).

[9] J. K. Freericks, V. M. Turkowski, and V. Zlatić, Phys. Rev. Lett. 97, 266408 (2006).

[10] P. Werner, T. Oka, and A. J. Millis, Phys. Rev. B 79, 035320 (2009).

[11] N. Tsuji, T. Oka, and H. Aoki, Phys. Rev. B 78, 235124 (2008).

[12] H. Lignier et al., Phys. Rev. Lett. 99, 220403 (2007).

[13] C.E. Creffield and T. S. Monteiro, Phys. Rev. Lett. 96, 210403 (2006).

[14] N. Tsuji, T. Oka, and H. Aoki, Phys. Rev. Lett. 103, 047403 (2009).

[15] M. Eckstein, M. Kollar, and P. Werner, Phys. Rev. Lett. 103, 056403 (2009).

[16] See, e.g., M. Rigol, V. Dunjko, and M. Olshanii, Nature (London) 452, 854 (2008), and references therein.

[17] To obtain $A(\omega, t)$ and $N(\omega, t)$ we Fourier- transform short time data using the Hanning function.

[18] The slice is taken as $f(\tilde{k}, t)=\left.f(\tilde{\boldsymbol{k}}, t)\right|_{\epsilon_{\tilde{k}}=-\cos \tilde{k}, v_{\tilde{k}}=\sin \tilde{k}}$.

[19] A. Eckardt and M. Holthaus, Europhys. Lett. 80, 50004 (2007).

[20] See, e.g., J. Guckenheimer and P. Holmes, Nonlinear Oscillations, Dynamical Systems, and Bifurcations of Vector Fields (Springer-Verlag, Berlin, 1983).

[21] R. Micnas, J. Ranninger, and S. Robaszkiewicz, Rev. Mod. Phys. 62, 113 (1990). 